\title{
An Overview of Psychogenic Non-Epileptic Seizures: Etiology, Diagnosis and Management
}

\author{
Ángela Milán-Tomás, Michelle Persyko, Martin del Campo, Colin M. Shapiro, \\ Karl Farcnik
}

\begin{abstract}
The purpose of this review is to provide an update of the research regarding the etiology, diagnosis and management of psychogenic non-epileptic seizures (PNES). A literature search using Pubmed, Ovid MEDLINE and EMBASE database was performed from 2000 up to August 2017. We have evaluated the different factors leading to PNES as well as the diagnostic approach and management of this disorder which continue to be very difficult. The coexistence of epilepsy and PNES poses special challenges and requires the coordinated efforts of the family physicians, psychiatrists, psychologists and neurologists. Although this condition has an overall poor prognosis, a multidisciplinary approach in the diagnosis and management of this disorder would likely improve the outcomes. We have proposed a diagnostic and treatment algorithm for PNES and suggested a national registry of patients suffering from this condition. The registry would contain data regarding treatment and outcomes to aid in the understanding of this entity.
\end{abstract}

RÉSUMÉ: Vue d'ensemble des crises psychogènes non-épileptiques: étiologie, diagnostic et prise en charge. L'objectif de cet article est de présenter une mise à jour des activités de recherche qui concernent l'étiologie, le diagnostic et la prise en charge des crises psychogènes non-épileptiques (CPNE). Pour ce faire, nous avons mené de 2000 à août 2017 une recherche bibliographique au moyen des bases de données suivantes : PubMed, Ovid MEDLINE et Embase. Nous avons procédé à l'évaluation des divers facteurs causant les CPNE, des approches diagnostiques et de la prise en charge de ce trouble, laquelle continue à être très difficile. La coexistence de l'épilepsie et des CPNE présente aussi des défis particuliers et exige des efforts coordonnés de la part des médecins de famille, des psychiatres, des psychologues et des neurologues traitants. Bien que les pronostics au sujet de cette condition soient généralement réservés, une approche multidisciplinaire dans l'établissement d'un diagnostic et la prise en charge des CPNE contribueraient probablement à améliorer l'évolution de l'état de santé des patients. À cet égard, nous avons proposé, en plus d'un registre national des patients atteints de ce trouble, un algorithme de diagnostic et de traitement pour les CPNE. À noter que ce registre contiendrait des données portant sur les modalités de traitement et leur résultat afin de favoriser la compréhension du cadre clinique des CPNE.

Keywords: Psychogenic non-epileptic seizures, PNES, Pseudoseizures, Non-epileptic attacks

doi:10.1017/cjn.2017.283

Can J Neurol Sci. 2018; 45: 130-136

\section{INTRODUCTION}

Psychogenic non-epileptic seizures (PNES) are a common phenomenon and there is no absolute consensus in defining this condition, so several terms have been used to describe these events over the years. ${ }^{1}$ A well-accepted notion is that they represent a sudden event characterized by paroxysmal changes in consciousness, movement and/or behavior that are similar to the ones seen in epileptic seizures. However, they are not associated with epileptiform activity as detected through video electroencephalography (VEEG) ${ }^{2}$ and there are psychologic underpinnings and causes that lead to the seizure. ${ }^{3}$

The term pseudo-seizures has been frequently used in the past ${ }^{4}$ but because it implies that the individual is "faking" it, alternative descriptors have been suggested. Accepted by some ${ }^{5,6}$ and adopted more widely ${ }^{1,7}$ the term PNES has been chosen as we feel it best describes these events.

The incidence of PNES has been estimated in 4.9/100,000/year ${ }^{8}$ and its prevalence varies widely between 2 and 33/ 100,000 population, ${ }^{9,10}$ probably due to variation in diagnostic criteria, the inherent complexity of PNES and misdiagnosis perhaps due to lack of proper training in this area. Although in the majority of cases the distinction between PNES and epileptic seizures can be made, ${ }^{11}$ to add more intricacy, PNES can be present in some patients with epilepsy. Some authors ${ }^{2,11,12}$ have reported that $5 \%$ to $15 \%$ of out-patient populations referred to epilepsy clinics and between $20 \%$ and $40 \%$ of in-patient populations admitted at epilepsy centers have PNES. In a recent retrospective study ${ }^{6}$ it was found that from 65 patients diagnosed with PNES, $11.1 \%$ also had epileptic seizures. In previous reports it has been described that $75 \%-80 \%$ of the patients with PNES are female. ${ }^{13}$ Although this disorder can occur at any age, Duncan et al. ${ }^{14}$ reported that the mean age of onset is 30.5 years ( $\mathrm{SD} \pm 13.7$ years) (see Table 2 ).

PNES have been known for at least 2,500 years, ${ }^{15}$ however, it continues to be a poorly understood disorder. The diagnosis of PNES is controversial and there is no definitive treatment approach. The overall prognosis of this condition is poor ${ }^{7}$ and it is

From the Department of Neurology, Sunnybrook Health Sciences Centre, University of Toronto, Toronto, Ontario, Canada (AM-T); Private Practice (MP); Department of Neurology, Toronto Western Hospital, University Health Network, Toronto, Ontario, Canada (MDC); Department of Psychiatry, Toronto Western Hospital, University Health Network, Toronto, Ontario, Canada (CMS, KF).

Received July 20, 2017. Final Revisions Submitted September 21, 2017. Date of ACCEPTANCE OCTOBER 15, 2017.

Correspondence to: A. Milan-Tomas, Sunnybrook Health Sciences Centre - Neurology, 2075 Bayview Ave, Toronto, ON, Canada M4N 3M5. Email: angela.milantomas@ sunnybrook.ca 
associated with a high level of disability and reduced quality of life (QOL). ${ }^{16}$

As far as we know, there is no data regarding the prevalence and incidence of this disorder in Canada; therefore, a database including the characteristics and outcomes of PNES would help clinicians to develop guidelines for the management of patients suffering from this condition (see Table 1 for the key points of this review).

\section{METHODS}

We present a comprehensive narrative review of PNES. To cover a wide range of topics related with PNES and add value to our manuscript we reviewed different sources including Pubmed, MEDLINE and EMBASE databases using OVID with literature pertaining PNES from the year 2000 and up to August 2017. We used the terms "PNES" as well as "pseudoseizures" and "psychogenic non-epileptic attacks" with the different following medical subheadings to identify relevant articles: "prevalence", "diagnosis", "management," "prognosis" and "outcomes".

The abstracts and bibliographies of relevant studies were reviewed to identify additional articles. We included only articles written in English and the evidence ranged from expert opinion to clinical trials, systematic reviews, meta-analyses and a Cochrane review was also included in this paper.

\section{PSYCHOPATHOLOGY OF PNES}

Various risk factors have been described in adults and children with PNES. In a study evaluating psychiatric comorbidities encountered in epilepsy monitoring units at Veterans Affairs Medical Centers in the USA, ${ }^{17}$ post-traumatic stress disorder (PTSD) preceded the diagnosis of PNES in 58\% of the patients. Major depression and alcohol abuse were common diagnoses in PNES and there is a well-documented association between chronic pain, psychiatric illness (especially depression, anxiety and personality disorders) and PNES (see Table 3). ${ }^{18,19}$ In children and adolescents parental discord or divorce, sexual or physical abuse, trauma, school difficulties, having epilepsy or family history of epilepsy among others are significantly associated factors. ${ }^{9,11,20,21}$

In a more comprehensive systematic review ${ }^{22}$ authors described different theoretical models of PNES: (1) PNES as a dissociative phenomenon, reflecting a defensive process that prevents individual from becoming overwhelmed by emotional adversity (seen for instance, as a somatic flashback in PTSD); (2) PNES as a "hardwired" reflex (tendency to have a defensive reflex as occur in other animals); (3) PNES as a learned behavior (maintained by positive and negative reinforcement); and (4) PNES as a disturbance of cognitive control (resulting and maintained by factors that increase activation of seizure programs). PNES has been classified in the Diagnostic and Statistical Manual of Mental Disorders, third revised edition (DSM-III-R), as a dissociative disorder. ${ }^{19}$ However, according to the DSM-5 and International Statistical Classification of Diseases and Related Health Problems (ICD)-10 manual, PNES is generally considered a conversion type of somatoform disorder. ${ }^{23,24}$ In a recent published study analyzing dissociative symptoms experienced by patients suffering from PNES; ${ }^{25}$ those with PNES compared with controls reported significantly more psychological and somatoform dissociative symptoms, trauma, perceived impact of trauma and post-traumatic symptoms. The relationship between traumatic brain injury and PNES has also been described, ${ }^{26}$ but the causality of this association should be further evaluated in prospective studies.
In addition, other disorders such as psychogenic movements disorders (PMD) have shown to share common risk factors (e.g. similar age and sex distribution; relatively frequent co-occurrence of psychiatric disorders or similar psychological profiles and high rates of chronic pain and other somatization disorders), ${ }^{27}$ complicating the understanding of the psychopathological process in PNES further. PNES and PMD may represent two ends of a continuum, where different clinical presentations explain referral to either the epilepsy or movement disorder specialists. ${ }^{27}$

\section{DIAGNOSIS}

The diagnosis of PNES is challenging and misdiagnosis of PNES as epilepsy can have deleterious consequences. ${ }^{28}$ Syncope, panic/anxiety, hyperventilation attacks, parasomnias, migraine with aura and transient ischemic attacks are some of the conditions that may give the appearance of both epileptic and non-epileptic seizures. $^{29,30}$ Sometimes accurate diagnosis is not established until 7-10 years or more after the onset of the disorder. ${ }^{28}$ Reasons that can contribute to this are a lack of knowledge by physicians with regards to PNES, negative connotation, and a lack of necessary diagnostic facilities. ${ }^{31,32}$

Some studies have noted that many signs that have been considered typical for PNES appear not to be specific as they can also be found in epileptic seizures, especially those which originate in the frontal lobe. ${ }^{33}$ Conversely, many features characteristic of epileptic seizures can also be present in PNES (i.e. tongue biting, urinary incontinence or eye closure).$^{34}$ Therefore, in primary care settings PNES should be suspected when a patient presents with seizures-like symptoms with resistance to antiepileptic drugs (AEDs) (though pharmacoresistant epilepsy is the other common cause of persistence seizures and reason for referral to an epilepsy center). ${ }^{35}$ The presence of some triggers may suggest also PNES. For instance, emotional or stress triggers, or if these events occur in special circumstances (in the presence of family members or physician's office). Patients who suffer from PNES usually have psychiatric comorbidities accompanying the syndrome (i.e. personality disorder, PTSD or depression/anxiety). ${ }^{19,20}$ However, it should be noted that the diagnosis of PNES cannot be made at this stage as no single clinical feature is pathognomonic of PNES. The International League Against Epilepsy (ILAE) Commission on Neuropsychobiology Non-epileptic Seizures Task Force was charged with developing a consensus on minimal requirements for diagnosis of non-epileptic events. ${ }^{3}$ They proposed a diagnostic level of certainty for PNES based in witnessed event, history and electroencephalogram (EEG) findings. Therefore, the level of certainty can be divided in: (1) Possible (if the event is selfreported by the patient or a witness and there is no epileptiform activity in a routine or sleep deprived EEG); (2) Probable (the event is described by a clinician who reviewed video recording and there is no epileptiform activity in the routine or sleep deprived EEG); (3) Clinically established (if the event is witnessed by a clinician experienced in diagnosis of seizure disorders and there is no epileptiform activity in the EEG during a typical event in which the semiology would make ictal epileptiform EEG activity expectable during equivalent epileptic seizure); and lastly, (4) Documented (when the event is witnessed by an experienced clinician in seizure disorders while on video EEG and there is no epileptiform activity immediately before, during or after the event captured on ictal video EEG with typical PNES semiology). ${ }^{3}$ 
Similar classification like the proposed by the ILAE for PNES, may be applicable to other psychogenic disorders such as psychogenic movement disorders. PNES has been labeled "psychogenic", implying that psychological factors lead to these events. However, it is not always easy to pinpoint the psychogenic mechanism involved as PNES is a complex disorder that requires a biopsychosocial conceptualization. ${ }^{36}$ Therefore, patient history, semiology and the use of other investigations such as neuroimaging may be needed in some cases.

Patients suspected of PNES should be referred to a neurologist who may perform further investigations, including a VEEG, which is considered the gold standard for the diagnosis of PNES. ${ }^{2}$ Although VEEG evaluation needs to be performed by an experienced clinician, recent studies have explored the use of artificial neural networks in the scoring of video recordings based on the signs and symptoms displayed (e.g. hypermotor versus akinetic or subjective symptoms). According to the authors, this technique may afford a measure of objectivity, in the differential diagnosis of PNES. ${ }^{37}$

Some criticisms have been made regarding the value of VEEG. For instance, events characterized by subjective sensory or autonomic manifestations require more comprehensive diagnostic procedures and the VEEG might be less sensitive in these cases. ${ }^{38}$ In one study evaluating the accuracy of epileptologists diagnosing PNES based on the video recording alone, ${ }^{38}$ authors found that there were some important factors affecting the rater's decision. For instance, the video or audio quality, as well as lack of intervention by bystanders, which were the most common reasons for considering the video below the minimum desirable standard to make a diagnosis. All five raters involved in this study were correct in predicting the diagnosis in 7 (30.4\%) of 23 cases. Of these, three had epileptic seizures and four PNES. However, for the diagnosis of other non-epileptic seizures, in addition to video and EEG data information about the prodromal symptoms and the results of monitoring other physiologic parameter, such as vital signs and ECG, might be necessary.

Another study evaluated the accuracy of discrimination between epileptic seizures and PNES before and after targeted video training among medical students. ${ }^{28}$ Authors showed that at 3 and 6 months after training the students achieved results similar to that of emergency medicine trainees, which suggest that videobased training is an effective tool to improve the accuracy of PNES diagnosis. ${ }^{28}$

Controversy also exists in the use of suggestive seizure induction as there is no unified protocol, definitive ethical agreement and no consensus on diagnostic utility. ${ }^{39}$ Hypnosis, intravenous application of saline, or even applying additional EEG-electrodes or photic stimulation could induce a psychogenic seizure as long as its effects are explained or implied convincingly. ${ }^{39}$ In a recent retrospective study including 122 patients with PNES, ${ }^{40}$ authors described that in about a quarter of all patients, no spontaneous PNES was recorded during VEEG, but one could be elicited during suggestive seizure induction. Therefore, the authors support the role of suggestive seizure induction in the diagnostic workup when PNES are suspected and no spontaneous events occur in the epilepsy monitoring unit.

A psychiatric and/or psychological evaluation is paramount as part of a comprehensive assessment strategy as well as to improve the outcomes. ${ }^{22,36}$ Interestingly, language-based techniques evaluating patient/physician interaction by conversation analysis may also be a useful tool in the differential diagnosis of PNES, especially when VEEG is not available. ${ }^{41}$

Laboratory studies might be helpful but are usually performed to exclude metabolic or toxic causes of seizures. Serum prolactin, creatine kinase and neuron-specific enolase have been evaluated in PNES; however, their limited discriminative power makes them unreliable. $^{42,43}$ A recent pilot study analyzing EEG spectral power in patients with $\mathrm{PNES}^{44}$ showed that desynchronization of $\beta$ power might be a marker of an upcoming non-epileptic attack. However, this needs to be reproduced in larger studies. In a systematic review evaluating biomarkers in the diagnosis of PNES (including neuroimaging findings, serum enzymes and hormones among others) authors concluded that no single biomarker successfully differentiates PNES from epileptic seizures. ${ }^{45}$

A prospective study analyzed the neuroanatomical correlates of PNES by using morphologic brain MRI measurements. ${ }^{46}$ Authors demonstrated that voxel-based morphometry and cortical thickness analyses in PNES patients were abnormal. They showed cortical atrophy of the motor and premotor regions in the right hemisphere and the cerebellum bilaterally. This observation was more pronounced in those with higher scores of depression, which favor the hypothesis of adaptive cortical-subcortical plasticity within these regions, giving rise to PNES (where PNES phenomenology is driven by the psychological factor interacting with specific biologic abnormalities). However, further studies are needed to clarify whether these changes are related to depression, other conversion disorders or to PNES per se.

Another study using positron emission tomography (PET) as functional neuroimaging, supported specific neurobiological dysfunction in patients with PNES. ${ }^{47}$ These patients exhibited significant hypometabolism within the right inferior parietal and central regions, and within the bilateral anterior cingulate cortex compared with controls. However, authors could not exclude that these observed changes may be related to patient's comorbidities, and these biomarkers are only used for research purposes at the moment.

Such studies may help in understanding the pathophysiology and refine the diagnosis of PNES in the future. Otherwise, incidental abnormalities can be occasionally seen on imaging, but they should not confound the diagnosis of PNES if this is established with the VEEG. We have proposed a diagnostic and management algorithm suggesting a multidisciplinary approach (see Figure 1).

\section{MANAGEMENT}

There is no standardized treatment strategy for PNES. Treatment recommendations are therefore based on case studies, small case series or anecdotal experience. ${ }^{13}$ Ideally, a team approach should be utilized to formulate a comprehensive treatment strategy. ${ }^{13}$ Patients with a suspicion of PNES should be referred by the family doctor to a neurologist who specializes in epilepsy for confirmation. These patients may be discharged from the care of the neurologist if there is no coexisting epilepsy. However, it is important that before discharge, patients have accepted their diagnosis and a transition to psychiatry has been established. ${ }^{48}$ This is the first step in the treatment pathway. Possible strategies for communicating the diagnosis of PNES include showing the patient a video-recording of the seizure, presenting the diagnosis as good news (i.e. the absence of epilepsy) and establishing that these seizures are not deliberate. ${ }^{49}$ However, one needs to be 


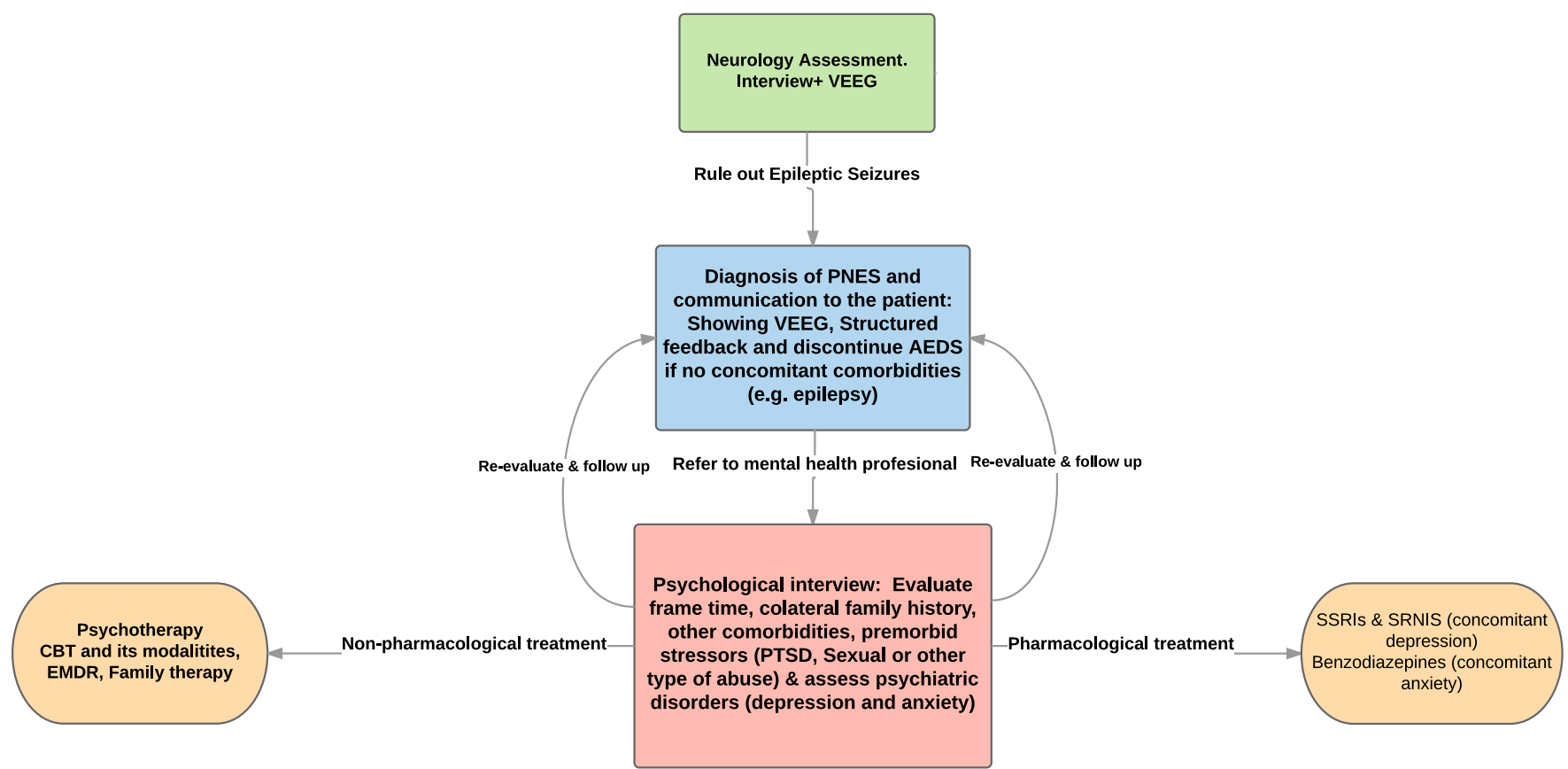

Figure 1: Diagnostic and Management Algorithm for psychogenic non-epileptic seizures (PNES). VEEG, video electroencephalogram; AEDS, antiepileptic drugs; PTSD, post-traumatic stress disorder; CBT, cognitive behavioral neurology; EMDR, eye movement desensitization and reprocessing; SSRIs, selective serotonin reuptake inhibitors; SRNIs, selective serotonin-norepinephrine reuptake inhibitors.

cautious as the diagnosis of PNES predicts a poor prognosis in many of the cases, so follow-ups and early interventions need to be put in place. Structured feedback and psychiatric consultation appears adequate to significantly reduce PNES frequency and improve aspects of QOL. ${ }^{36}$ A clinical trial published recently showed that structured feedback interventions, which may contribute to better acceptance of the PNES diagnosis, led to a greater likelihood of seeking formal mental health intervention, reduction in PNES events and improvements in QOL. Furthermore, the addition of weekly phone contact also led to mood improvement. ${ }^{36}$

Current treatments include the use of cognitive behavioral therapy (CBT) by using desensitization, and exposure plus response prevention to modify anxiety and allow adaptive responses. $^{30}$ Other special forms of CBT such as hypnotic abreaction to try to discover how the patients feel during seizures have been also recommended. ${ }^{32}$ Prolonged exposure (PE) therapy is also another form of CBT that specifically targets the effects of PTSD and was used in a trial of 16 patients with PNES and PTSD. ${ }^{50}$ In this trial authors showed that although a diagnois of PTSD does not explain the development of PNES in all patients,

\section{Table 1: Key points}

\begin{tabular}{l} 
Psychogenic non-epileptic seizures (PNES) are a common phenomenon which is \\
poorly understood and underdiagnosed \\
\hline Epilepsy and psychiatric co-morbidities are common in this patient population \\
\hline Video-EEG is considered the gold standard for the diagnosis of PNES \\
\hline Psychiatric evaluation should be performed after the diagnosis of PNES has been \\
established as a part of the management strategy \\
\hline $\begin{array}{l}\text { Current treatments include the use of cognitive behavioural therapy (CBT), hypnosis, } \\
\text { family therapy and medications, which can be useful when other comorbidities such } \\
\text { as depression are present }\end{array}$
\end{tabular}

applying a disorder-specific treatment (PE therapy for PTSD) can be an effective way of treating PNES in patients who have been traumatized and carry a diagnosis of PTSD.

In an open pilot trial, CBT was found to reduce PNES frequency and improve psychosocial functioning after 12 sessions of treatment. ${ }^{51} \mathrm{~A}$ meta-analysis evaluating the prevalence of seizure reduction in psychological treatments for PNES, authors showed that $82 \%$ of those completing psychotherapy reported a reduction in seizure frequency of $\geq 50 \% .{ }^{52}$ Goldstein et al. ${ }^{53}$ proposed a multicenter randomized controlled trial of CBT for PNES evaluating the cost effectiveness of this intervention and its generalizability which could help us to make further recommendations in the management of patients with PNES. Furthermore, we need to take into account that mental health resources as well as specific protocols approaching the diagnosis and management of PNES might not be available in all neurology centers where the diagnosis of PNES is made. ${ }^{54}$

A recent meta-analysis reviewing 13 studies that included CBT, psychodynamic therapy, paradoxical intention therapy, mindfulness, psychoeducation and eclectic interventions in patients with PNES, highlighted the potential for psychological interventions as a favorable alternative to the current lack of

Table 2: Epidemiology of psychogenic non-epileptic seizures (PNES)

\begin{tabular}{l}
\hline Its prevalence is approximately 2-33/100.000 population ${ }^{9,10}$ \\
\hline The mean onset of PNES is 30.5 years $( \pm 13.7)^{14}$ \\
\hline $75 \%-80 \%$ of patients with PNES are women ${ }^{13}$ \\
\hline $\begin{array}{l}\text { Concomitant epilepsy and PNES: } 5 \%-10 \% \text { in a general population versus } 20 \%-40 \% \text { in } \\
\text { epilepsy clinics }\end{array}$
\end{tabular}


Table 3: Common factors and comorbidities in psychogenic non-epileptic seizures (PNES)

Physical/sexual abuse, trauma or a family member with epilepsy

Alcohol abuse

Chronic pain

Other psychiatric illness (i.e. depression, post-traumatic disorder and anxiety)

treatment options offered to people with PNES. ${ }^{52}$ However, in a Cochrane review that included 12 randomized clinical trials of CBT in patients suffering from PNES, ${ }^{55}$ authors showed that many of these studies have multiple biases and the overall evidence for the main outcome of reducing seizures as a result of treatment was not considered reliable. Therefore, further research in this field needs to be done.

Pharmacotherapy should be considered especially when major mood disorders such as depression, panic disorder or psychosis are present ${ }^{30}$. LaFrance et al. ${ }^{56}$ suggested that a selective serotonin reuptake inhibitor or related compounds may be useful. However, there is insufficient evidence to recommend routine treatment with antidepressant medication as a method of reducing PNES. ${ }^{57} \mathrm{An}$ open-label prospective study evaluated patients with PNES and anxious-depressive symptoms treated with venlafaxine for 5 months. ${ }^{58}$ The authors showed that venlafaxine reduced by $50 \%$ the number of seizure like events as well as depressive and anxiety symptoms based on different scales.

In the absence of epilepsy, an important first step once the diagnosis of PNES is made is to discontinue the AEDs as they may have associated side effects. ${ }^{59}$ However, many patients with PNES in whom epilepsy has been excluded remain on AEDs. ${ }^{59}$ In a study cohort was shown that a baseline history of psychological trauma was a predictor for continuation of AED prescription 5-10 years after the diagnosis of PNES was made. ${ }^{14}$ Of note, the continuation of AEDs might be considered to manage other comorbidities (e.g. neuropathic pain and psychiatric disorders such as depression or bipolar disorder). ${ }^{11,12}$ Part of PNES management is to have further neurology follow up visits to assess the overall progress of this condition as well as if necessary to explain again the diagnosis and supervise the AED withdrawal in the transition to mental health professional evaluation.

\section{PROGNOSIS}

As commented above, communicating the diagnosis to the patient may impact upon the outcomes of this condition and it has been shown that a standardized diagnostic approach and feedback process may reduce event frequency. ${ }^{36}$ Other factors such as educational status and being accompanied to the first clinic visit have also been described as favorable predictors of outcomes. ${ }^{60}$ However, prospective studies evaluating the prognosis of these patients are controversial and some had shown poor outcomes despite an effective communication strategy. ${ }^{61,62}$ In a recent study assessing standard medical care for PNES across Brazilian epilepsy centers, none of the centers had a particular protocol with rigorous guidelines to provide the diagnosis and treat PNES. ${ }^{63}$ This probably represents just an example of the need for guidelines regarding management of PNES in different countries. The physician presenting the diagnosis must be compassionate

\section{Table 4: Unanswered questions and possible future directions}

There is no standardized treatment strategy for PNES
$\begin{aligned} & \text { Treatment recommendations are based on case studies, small case series or anecdotal } \\ & \text { experience }\end{aligned}$
A national registry of patients with PNES will help us to improve the management and
outcomes of PNES

PNES, psychogenic non-epileptic seizures.

and remember that most of the patients with this problem are not intentionally faking. Patients who accept their diagnosis and follow through with therapy are more likely to experience a successful outcome. However, at least 50\%-70\% of the patients will have some improvement but will not be seizure free ${ }^{64}$ and about one-quarter of the patients develop chronic PNES. ${ }^{13}$

Psychological factors (including anxiety, depression and illness perceptions) are a stronger predictor of health-related QoL than condition-related and demographic variables in epilepsy and PNES. Therefore, different studies have shown the importance of addressing patients' beliefs about their condition. ${ }^{65}$

Unfortunately, those patients with only improvement, but not cessation of PNES, continue to experience significant difficulties. Some of these difficulties include unemployment, depression, persistent visits to the emergency department and reduced QOL in both, patients and caregivers. ${ }^{66}$ Karakis et al. ${ }^{66}$ showed that QOL scores were significantly worse for PNES than for patients with epileptic seizures and were mainly linked to depression levels. In addition to the social and psychological impact, the financial burden of this disorder is high. ${ }^{10}$ It is reported that over $50 \%$ of PNES patients are disabled. ${ }^{2}$ An observational study suggested increased mortality in these patients. ${ }^{67}$ Although the overall outcome is poor in PNES, ${ }^{64}$ some factors are associated with worse prognosis. Some examples are delay in the diagnosis, psychiatric hospitalizations, PTSD, sexual and/or physical abuse and psychiatric comorbidities such as depression or anxiety. Moreover, being single, specific coping strategies (escape-avoidance coping style), family functioning (unsupportive or uninterested family environment) and low socioeconomic status also impact negatively the prognosis of PNES. ${ }^{16,68,69}$ On the other hand, there are some factors that can predict treatment adherence and therefore improve the outcomes of these patients. A retrospective observational study showed that PNES patients who are married or have a live-in partner and subjects without cognitive impairment were more likely to be adherent. ${ }^{30}$

The prognosis in children with PNES might be better, with close to $70 \%$ achieving remission. ${ }^{70,71}$ However, this needs to be read with caution as the results are based in small retrospective studies, so bigger cohorts and prospective research studies are needed in this population. Psychiatric illness (especially depression) has been shown to be frequent in this population. ${ }^{21} \mathrm{~A}$ delay in the diagnosis may result in iatrogenic complications. Therefore, identifying PNES in childhood and adolescence should be considered of the utmost importance. ${ }^{20}$

\section{DISCUSSION}

PNES continues to be both a diagnostic and treatment challenge. It is associated with significant morbidity that it is comparable with a population with epilepsy and according to some authors may even be associated with a worse outcome. 
Furthermore, at this time there is no consensus on the management of PNES; overall strategies are based on small case series. A significant percentage of patients with PNES continue to manifest symptoms which profoundly affect the individual.

We have proposed a diagnostic and treatment algorithm suggesting a multidisciplinary approach to probably improve the outcomes of patients suffering from PNES. A national registry of patients with PNES, their treatment and outcomes may help improve the understanding of this entity and establish more effective treatment strategies and guidelines through coordinated research and "big data" collaborations across the country (see Table 4 for unanswered questions and future directions).

The coexistence of epilepsy and PNES poses special challenges and requires the coordinated efforts of the psychiatrist and the neurologist, particularly when seizure threshold-lowering drugs need to be administered. The use of CBT and other modalities of psychotherapy should be further evaluated in bigger cohort of patients.

\section{ACKNOWLEDGMENTS}

ÁM-T acknowledges the financial support received by Dr. KF and Dr. CMS for her research fellowship in sleep medicine at the psychiatry department in Toronto Western Hospital. ÁM-T also thanks Dr. KF, Dr. CMS, Dr. MD, and MP for their intellectual input, writing assistance, language editing, and proofreading.

\section{Disclosures}

ÁM-T, MP, MdC, CMS and KF do not have any disclosures.

\section{Statement OF AUthorship}

Authors contributed equally to this work.

\section{REFERENCES}

1. Brigo F, Igwe SC, Ausserer H, et al. Terminology of psychogenic nonepileptic seizures. Epilepsia. 2015;56(3):e21-e25.

2. Reuber M, Mayor R. Recent progress in the understanding and treatment of nonepileptic seizures. Curr Opin Psychiatry. 2012;25:244-50.

3. LaFrance WC Jr., Baker GA, Duncan R, Goldstein LH, Reuber M. Minimum requirements for the diagnosis of psychogenic nonepileptic seizures: a staged approach: a report from the International League Against Epilepsy Nonepileptic Seizures Task Force. Epilepsia. 2013;54(11):2005-18.

4. Glaser GH. Epilepsy, hysteria and "Possession". A historical essay. J Nerv Metn Dis. 1978;166(4):268-74.

5. Bodde NM, van der Kruijs SJ, Ijff DM, et al. Subgroup classification in patients with psychogenic non-epileptic seizures. Epilepsy Behav. 2013;26(3):279-89.

6. Wadwekar V, Nair PP, Murgai A, et al. Semiologic classification of psychogenic non epileptic seizures (PNES) based on video EEG analysis: do we need new classification systems? Seizure. 2014;23(3):222-6.

7. Tannemaat MR, van Dijk JG. The terminology of psychogenic nonepileptic seizures: a historical perspective. Epilepsia. 2015;56(6): 978-9.

8. Duncan R, Razvi S, Mulhern S. Newly presenting psychogenic nonepileptic seizures: incidence, population characteristics, and early outcome from a prospective audit of a first seizure clinic. Epilepsy Behav. 2011;20(2):308-11.

9. Benbadis SR, Allen Hauser W. An estimate of the prevalence of psychogenic non-epileptic seizures. Seizure. 2000;9(4):280-1.

10. Sahaya K, Dholakia SA, Lardizabal D, Sahota PK. Opinion survey of health care providers towards psychogenic non epileptic seizures. Clin Neurol Neurosurg. 2012;114(10):1304-7.
11. Asadi-Pooya AA, Emami M. Demographic and clinical manifestations of psychogenic non-epileptic seizures: the impact of co-existing epilepsy in patients or their family members. Epilepsy Behav. 2013;27(1):1-3.

12. Benbadis SR, Agrawal V, Tatum WO. How many patients with psychogenic non-epileptic seizures also have epilepsy? Neurology. 2001;57:915-7.

13. Bodde NM, Brooks JL, Baker GA, et al. Psychogenic non-epileptic seizures-diagnostic issues: a critical review. Clin Neurol Neurosurg. 2009;111(1):1-9.

14. Duncan R, Graham CD, Oto M, Russell A, et al. Primary and secondary care attendance, anticonvulsant and antidepressant use and psychiatric contact 5-10 years after diagnosis in 188 patients with psychogenic non-epileptic seizures. J Neurol Neurosurg Psychiatry. 2014;85(9):954-8.

15. Veith I. Hysteria: The History of a Disease. Chicago, IL: University of Chicago Press; 1965.

16. Myers L, Lancman M, Laban-Grant O, et al. Psychogenic nonepileptic seizures: predisposing factors to diminished quality of life. Epilepsy Behav. 2012;25(3):358-62.

17. Salinsky M, Evrard C, Storzbach D, Pugh MJ. Psychiatric comorbidity in veterans with psychogenic seizures. Epilepsy Behav. 2012;25(3):345-9.

18. Benbadis SR. A spell in the epilepsy clinic and a history of "chronic pain" or "fibromyalgia" independently predict a diagnosis of psychogenic seizures. Epilepsy Behav. 2005;6:264-5.

19. Bowman ES, Markand ON. Psychodynamics and psychiatric diagnoses of pseudoseizure subjects. Am J Psychiatry. 1996; 153(1):57-63.

20. Say GN, Tasdemir HA, Akbas S, et al. Self-esteem and psychiatric features of Turkish adolescents with psychogenic non-epileptic seizures: a comparative study with epilepsy and healthy control groups. Int J Psychiatry Med. 2014;47(1):41-53.

21. Vincentiis S, Valente KD, Thomé-Souza S, et al. Risk factors for psychogenic nonepileptic seizures in children and adolescents with epilepsy. Epilepsy Behav. 2006;8(1):294-8.

22. Brown RJ, Reuber M. Towards an integrative theory of psychogenic non-epileptic seizures (PNES). Clin Psychol Rev. 2016;47: 55-70.

23. American Psychiatric Association. Diagnostic and Statistical Manual of Mental Disorders - Fifth Edition. Arlington, VA: American Psychiatric Association; 2013.

24. World Health Organisation (WHO). ICD-10, Available from www. who.int/classifications/icd/en/bluebook.pdf (5 April 2017).

25. Pick S, Mellers JD, Goldstein LH. Dissociation in patients with dissociative seizures: relationships with trauma and seizure symptoms. Psychol Med. 2017;47(7):1215-29.

26. Salinsky M, Storzbach D, Goy E, Evrard C. Traumatic brain injury and psychogenic seizures in veterans. J Head Trauma Rehabil. 2015;30(1):E65-E70.

27. Erro R, Brigo F, Trinka E, Turri G, Edwards MJ, Tinazzi M. Psychogenic nonepileptic seizures and movement disorders: a comparative review. Neurol Clin Pract. 2016;6(2):138-49.

28. Seneviratne U, Ding C, Bower S, et al. Video-based training improves the accuracy of seizure diagnosis. J Neurol Neurosurg Psychiatry. 2014;85(4):466-70.

29. Brodtkorb E. Common imitators of epilepsy. Acta Neurol Scand Suppl. 2013;(196):5-10.

30. Baslet G, Seshadri A, Bermeo-Ovalle A, et al. Psychogenic nonepileptic seizures: an updated primer. Psychosomatics. 2016; 57(1):1-17.

31. MacDonald T, Hill A, Phan T, et al. Psychiatry versus general physicians: who is better at differentiating epileptic from psychogenic non-epileptic seizures? Australas Psychiatry. 2012;20(5):379-83.

32. Bodde NM, Brooks JL, Baker GA, et al. Psychogenic non-epileptic seizures-definition, etiology, treatment and prognostic issues: a critical review. Seizure. 2009;18(8):543-53.

33. Pillai JA, Haut SR. Patients with epilepsy and psychogenic nonepileptic seizures: an inpatient video-EEG monitoring study. Seizure. 2012;21(1):24-7.

34. Brigo F, Ausserer H, Nardone R, et al. Clinical utility of ictal eyes closure in the differential diagnosis between epileptic seizures and psychogenic events. Epilepsy Res. 2013;104(1-2):1-10. 
35. Benbadis, SR. (Updated June 15, 2017). Psychogenic nonepileptic seizures. Available at https://emedicine.medscape.com/article/ 1184694-overview.

36. Drane DL, LaRoche SM, Ganesh GA, et al. A standardized diagnostic approach and ongoing feedback improves outcome in psychogenic nonepileptic seizures. Epilepsy Behav. 2016;54:34-9.

37. Magaudda A, Laganà A, Calamuneri $\mathrm{A}$, et al. Validation of a novel classification model of psychogenic nonepileptic seizures by video-EEG analysis and a machine learning approach. Epilepsy Behav. 2016;60:197-201.

38. Erba G, Giussani G, Juersivich A, et al. The semiology of psychogenic nonepileptic seizures revisited: Can video alone predict the diagnosis? Preliminary data from a prospective feasibility study. Epilepsia. 2016;57(5):777-85.

39. Popkirov S, Grönheit W, Wellmer J. A systematic review of suggestive seizure induction for the diagnosis of psychogenic nonepileptic seizures. Seizure. 2015;31:124-32.

40. Popkirov S, Jungilligens J, Grönheit W, Wellmer J. Diagnosing psychogenic nonepileptic seizures: video-EEG monitoring, suggestive seizure induction and diagnostic certainty. Epilepsy Behav. 2017;73:54-8.

41. Cornaggia CM, Gugliotta SC, Magaudda A, Alfa R, Beghi M, Polita M. Conversation analysis in the differential diagnosis of Italian patients with epileptic or psychogenic non-epileptic seizures: a blind prospective study. Epilepsy Behav. 2012;25(4):598-604.

42. Willert C, Spitzer C, Kusserow S, Runge U. Serum neuron-specific enolase, prolactin, and creatine kinase after epileptic and psychogenic non-epileptic seizures. Acta Neurol Scand. 2004; 109(5):318-23.

43. Nass RD, Sassen R, Elger CE, Surges R. The role of postictal laboratory blood analyses in the diagnosis and prognosis of seizures. Seizure. 2017;47:51-65.

44. Meppelink AM, Pareés I, Beudel M, et al. Spectral power changes prior to psychogenic non-epileptic seizures: a pilot study. J Neurol Neurosurg Psychiatry. 2017;88(2):190-2.

45. Sundararajan T, Tesar GE, Jimenez XF. Biomarkers in the diagnosis and study of psychogenic nonepileptic seizures: a systematic review. Seizure. 2016;35:11-22.

46. Labate A, Cerasa A, Mula M, et al. Neuroanatomic correlates of psychogenic nonepileptic seizures: a cortical thickness and VBM study. Epilepsia. 2012;53(2):377-85.

47. Arthuis M, Micoulaud-Franchi JA, Bartolomei F, et al. Resting cortical PET metabolic changes in psychogenic non-epileptic seizures (PNES). J Neurol Neurosurg Psychiatry. 2015;86(10): 1106-1112.

48. Reuber M, Elger CE. Psychogenic nonepileptic seizures: review and update. Epilepsy Behav. 2003;4(3):205-16.

49. Marie Gillig P. Psychogenic nonepileptic seizures. Innov Clin Neurosci. 2013;10(11-12):15-8.

50. Myers L, Vaidya-Mathur U, Lancman M. Prolonged exposure therapy for thetreatment of patients diagnosed with psychogenic non-epileptic seizures(PNES) and post-traumatic stress disorder (PTSD). Epilepsy Behav. 2017;66:86-92.

51. Goldstein LH, Deale AC, Mitchell-O'Malley SJ, et al. An evaluation of cognitive behavioral therapy as a treatment for dissociative seizures: a pilot study. Cogn Behav Neurol. 2004;17(1):41-9.

52. Carlson P, Nicholson Perry K. Psychological interventions for psychogenic non-epileptic seizures: a meta-analysis. Seizure. 2017;45:142-50.

53. Goldstein LH, Mellers JD, Landau S, et al. Cognitive behavioural therapy vs standardised medical care for adults with dissociative non-epileptic seizures (CODES): a multicentre randomised controlled trial protocol. BMC Neurol. 2015;15:98.

54. Valente KD, De Paola L, Palmini A, et al. The approach to patients with psychogenic nonepileptic seizures in epilepsy surgery centers regarding diagnosis, treatment, and education. Epilepsy Behav. 2017;68:78-83.

55. Martlew J, Pulman J, Marson AG. Psychological and behavioural treatments for adults with non-epileptic attack disorder. Cochrane Database Syst Rev. 2014;(2):CD006370.

56. LaFrance WC Jr., Baird GL, Barry JJ, et al. NES Treatment Trial (NEST-T) Consortium. Multicenter pilot treatment trial for psychogenic nonepileptic seizures: a randomized clinical trial. JAMA Psychiatry. 2014;71(9):997-1005.

57. Bravo TP, Hoffman-Snyder CR, Wellik KE, et al. The effect of selective serotonin reuptake inhibitors on the frequency of psychogenic nonepileptic seizures: a critically appraised topic. Neurologist. 2013;19(1):30-3.

58. Pintor L, Baillés E, Matrai S, et al. Efficiency of venlafaxine in patients with psychogenic nonepileptic seizures and anxiety and/ or depressive disorders. J Neuropsychiatry Clin Neurosci. 2010; 22(4):401-8.

59. Duncan R. The withdrawal of antiepileptic drugs in patients with non-epileptic seizures: safety considerations. Expert Opin Drug Saf. 2006;5(5):609-13

60. Arain AM, Hamadani AM, Islam S, Abou-Khalil BW. Predictors of early seizure remission after diagnosis of psychogenic nonepileptic seizures. Epilepsy Behav. 2007;11(3):409-12.

61. Mayor R, Brown RJ, Cock H, et al. Short-term outcome of psychogenic non-epileptic seizures after communication of the diagnosis. Epilepsy Behav. 2012;25(4):676-81.

62. Salinsky M, Storzbach D, Goy E, Kellogg M, Boudreau E. Health care utilization following diagnosis of psychogenic nonepileptic seizures. Epilepsy Behav. 2016;60:107-11.

63. Valente KD, De Paola L, Palmini A, et al. The approach to patients with psychogenic nonepileptic seizures in epilepsy surgery centers regarding diagnosis, treatment, and education. Epilepsy Behav. 2017;68:78-83.

64. Iriarte J, Parra J, Urrestarazu E, Kuyk J. Controversies in the diagnosis and management of psychogenic pseudoseizures. Epilepsy Behav. 2003;4(3):354-9.

65. Rawlings GH, Brown I, Reuber M. Predictors of health-related quality of life in patients with epilepsy and psychogenic nonepileptic seizures. Epilepsy Behav. 2017;68:153-8.

66. Karakis I, Montouris GD, Piperidou C, et al. Patient and caregiver quality of life in psychogenic non-epileptic seizures compared to epileptic seizures. Seizure. 2014;23(1):47-54.

67. Duncan R, Oto M, Wainman-Lefley J. Mortality in a cohort of patients with psychogenic non-epileptic seizures. J Neurol Neurosurg Psychiatry. 2012;83(7):761-2.

68. Baslet G, Prensky E. Initial treatment retention in psychogenic nonepileptic seizures. J Neuropsychiatry Clin Neurosci. 2013;25(1): 63-67.

69. Jones B, Reuber M, Norman P. Correlates of health-related quality of life in adults with psychogenic nonepileptic seizures: a systematic review. Epilepsia. 2016;57(2):171-81.

70. Irwin K, Edwards M, Robinson R. Psychogenic non-epileptic seizures: management and prognosis. Arch Dis Child. 2000; 82(6):474-478

71. Yi YY, Kim HD, Lee JS, Cheon KA, Kang HC. Psychological problems and clinical outcomes of children with psychogenic non-epileptic seizures. Yonsei Med J. 2014;55(6):1556-61. 